\title{
If you cannot move, send messengers: how cells organize space
}

\author{
Peter Nick ${ }^{1}$
}

(C) Springer-Verlag Wien 2016

Life is cellular-the description of cork "cells" by Robert Hooke 350 years ago turned out to be a general principle. Cells allow to delineate an internal space that is defended against fluctuations of the external world. The resilience of this internal homeostasis will decide, which environments can be conquered. However, adaptation of the interior is not the only strategy employed-multicellular organisms have achieved the ability to organize the space surrounding individual cells in a manner that adverse fluctuations can be buffered. There are two principle approaches to achieve this: Either cells differentiate (meaning that they establish, in a more or less stable manner, a different type of homeostasis) and move into different locations. Alternatively, cells that are not able to move (for instance plant cells) have to send out messengers that do this job. These messengers are usually of chemical nature - either macromolecules such as proteins or RNA species, or specific (i.e., secondary) metabolites. Three contributions in the current issue highlight different aspects of the second approach and show the activity of quite diverse mechanisms of transport, stimulating the general question, how these mechanisms are controlled in space and time.

The review by Becker and Ehlers (2016) in the current issue considers the differentiation of leaf whorls into flower organs. The identity of sepals, petals, anthers, and carpels is controlled by dimeric complexes of transcription factors that steer the differentiation of the developing leaf primordium. The study of homeotic mutants of flower patterning, such as those already described by Johann Wolfgang v. Goethe more

\section{Peter Nick}

peter.nick@kit.edu

1 Molecular Cell Biology, Karlsruhe Institute of Technology, Karlsruhe, Germany than two centuries ago, culminated in the ingenious $\mathrm{ABC}$ model, proposing that flower organ identity is defined by homo- or heterodimeric interaction of a limited number of genetic switches. What at first sight looked like a fairly cell autonomous process, later turned out to be strongly controlled by transport of signals: Secretion of peptides and proteins controls the balance between stem cell maintenance and differentiation in the apical meristem. Far-distance signals are generated in the leaves in a complex response to day length and are then translocated as florigen to the apical meristem. And, floral identity is not cell autonomous either, but controlled by intensive exchange of transcriptional regulators. Flowering thus emerges as a process, where numerous cells from quite different locations in the plant communicate by macromolecular signals and channel cell fate in a very specific region of the apical meristem in a manner that will ensure efficient gene flow for the entire organism. This shifts abundance, location, and gating of plasmodesmata into the center of pattern formation (Burch-Smith et al. 2011).

A very specific and fundamental aspect of intracellular messengers is the interaction between the eukaryotic nucleus and the semi-autonomous organelles such as mitochondria and plastids that represent formerly independent prokaryotic organisms which had been "domesticated." Although some of the gene products required for the specific functions of these organelles are still encoded in the organelle genome, most genes had been transferred to the nucleus during the long history of domestication. This means that most proteins have to be imported and that this import has to be regulated depending on the demand in the organelle. The review by Paić and Fulgosi (2016) in the current issue highlights the role of specific immunophilins for maintenance and repair of plastid functionality. This group of proteins is as widespread as it has remained enigmatic. Although found in all life forms, their extreme functional versatility has left them somehow 
nebulous. The core function seems to be the ability to isomerize peptide bonds containing proline, which often represents the bottleneck for protein folding. A particularly large diversity of these proteins - that normally convey a broad range in the cytoplasm - can be found in the thylakoid lumen of chloroplasts, one of the most challenged compartments, since light-dependent water splitting and activation of free electrons create an extremely dangerous and demanding environment. Here, continuous and flexible repair and reconstitution of complexes is vital to maintain functionality. This leads to the interesting question, how the abundance and activity of these immunophilins in the thylakoid lumen is regulated depending on the status of the chloroplast. Again, extensive transport, this time from the cytoplasm through two membranes into the thylakoid lumen, is required, probably involving extensive unfolding and refolding.

Although transport of proteins and peptides seems to be a general mechanism of inter- and intracellular interaction, it should be kept in mind that also specific small molecules can be transported over considerable distance. This is concluded from the work by Amrehn et al. (2016) in the current issue that dissects the cellular aspects of secondary metabolites found in the Asteraceae, the sesquiterpene lactone. This group of compounds has recently acquired considerable attention in the context of the Nobel Prize for medicine awarded for the discovery and exploitation of the Malaria remedy artemisinin. These compounds are produced in a peculiar type of glands called capitate glandular trichomes, where the bioactive product is collected in a subcuticular compartment (i.e., outside of the plasma membrane). After the authors had generated a careful structural model of this secretory tissue in sunflower (Amrehn et al. 2014), they now ventured to dissect the functional relationships of its components. The classical view on these metabolites assumed that they are synthetised in and secreted exclusively from the terminal cell. The presence of cell wall protruberances characteristic of transfer cells (Talbot and McCurdy 2002) supports secretion from the terminal cells. To address, whether possibly additional cells participate in the synthesis of these metabolites, authors use the approach to immunolabel one of the key enzyme, germacrane oxidase, and then to detect the signal either by fluorescence microscopy or, after immunogold labelling, by transmission electron microscopy. The authors can show that the enzyme is linked with the smooth ER, and they find, unexpectedly, that it is not only present in the terminal cell, but also in the subapical cells of the stalk. This leads to the question, how the product is translocated to the subcuticular compartment, which is located in quite some distance. Since plasmodesmata between the cells of the gland are scarce, they arrive at the conclusion that transport is likely to be apoplastic.

\section{Compliance with ethical standards}

Conflict of interest The author declares that there is no conflict of interest.

\section{References}

Amrehn E, Aschenbrenner AK, Heller AR, Spring O (2016) Localization of sesquiterpene lactone biosynthesis in cells of capitate glandular trichomes of Helianthus annuus (Asteraceae)

Amrehn E, Heller A, Spring O (2014) Capitate glandular trichomes of Helianthus annuus (Asteraceae): ultrastructure and cytological development. Protoplasma 251:161-167

Becker A, Ehlers K (2016) Arabidopsis flower development—of protein complexes, targets, and transport. Protoplasma, current issue

Burch-Smith TM, Stonebloom S, Xu M, Zambryski PC (2011) Plasmodesmata during development: re-examination of the importance of primary, secondary, and branched plasmodesmata structure versus function. Protoplasma 248:61-74

Paić A, Fulgosi H (2016) Chloroplast immunophilins. Protoplasma, current issue

Talbot ME, Offler CE, McCurdy DW (2002) Transfer cell wall architecture: a contribution towards understanding localized wall deposition. Protoplasma 219:197-209 